\title{
TeV Scale Seesaw Model, Scalar Dark Matter and Electroweak Vacuum Stability
}

\author{
Srubabati Goswami* \\ Theoretical Physics Division, Physical Research Laboratory, Ahmedabad - 380009, India \\ E-mail: sruba@prl.res.in \\ Ila Garg \\ Department of Physics, Indian Institute of Technology Bombay, Powai, Mumbai 400 076, India \\ E-mail: ila.garg@iitb.ac.in
}

\section{Vishnudath K. N.}

Theoretical Physics Division, Physical Research Laboratory, Ahmedabad - 380009, India Discipline of Physics, Indian Institute of Technology, Gandhinagar - 382355, India

E-mail: vishnudath@prl.res.in

\section{Najimuddin Khan}

Centre for High Energy Physics, Indian Institute of Science, C. V. Raman Avenue, Bangalore 560012, India

E-mail: khanphysics.123@gmail.com

\begin{abstract}
We consider a singlet extension of the standard model in which the active light neutrino masses are generated via the $\mathrm{TeV}$ scale inverse seesaw mechanism whereas a singlet scalar is introduced as the candidate for dark matter. We show that although these two sectors are disconnected at low energy, the coupling constants of both the sectors get correlated at high energy scale by the constraints coming from the perturbativity and stability/metastability of the electroweak vacuum.
\end{abstract}

The 39th International Conference on High Energy Physics (ICHEP2018)

4-11 July, 2018

Seoul, Korea

${ }^{*}$ Speaker. 


\section{Inverse Seesaw Mechanism, Scalar Dark Matter and Vacuum Stability}

It is well known that the electroweak vacuum of the Standard Model (SM) is in the metastable region. We have studied the stability of the electroweak vacuum in the context of $\mathrm{TeV}$ scale inverse seesaw extended with a scalar singlet dark matter. Thus, the additional particles are three right handed neutrinos $N_{R}$, three singlet fermions $v_{S}$ and a singlet scalar $S . N_{R}$ and $v_{s}$ have opposite lepton numbers and the scalar $S$ is odd under a $Z_{2}$ symmetry $S \rightarrow-S$. We analyze the interplay between the contribution of the extra singlet scalar and the singlet fermions to the EW vacuum stability. The coupling constants in these two seemingly disconnected sectors can be correlated at high energy by the vacuum stability/metastability and perturbativity constraints.

The model parameters have been fitted with the neutrino oscillation data and non-unitarity constraints on the PMNS matrix. We have taken the heavy neutrino masses of order up to a few $\mathrm{TeV}$. The extra $Z_{2}$ symmetry ensures that the scalar particle serves as a viable dark matter candidate. For the masses of new fermions from $200 \mathrm{GeV}$ to a few $\mathrm{TeV}$, the annihilation cross section to the extra fermions is very small for dark matter mass, $M_{D M} \sim \mathscr{O}(1-2) \mathrm{TeV}$. For $M_{D M}>>M_{t}$, one can approximately write $M_{D M}$ $\sim 3300 \kappa \mathrm{GeV}$. Here, $\kappa$ is the quartic scalar coupling between the SM Higgs and the extra singlet scalar The theory violates perturbativity before the Planck scale for $M_{D M} \gtrsim 2.5 \mathrm{TeV}$. Values of $\kappa<0.16$ are disallowed by LUX-2016 data along with the relic density constraints.

The presence of the fermionic Yukawa couplings drives the vacuum more towards instabil-

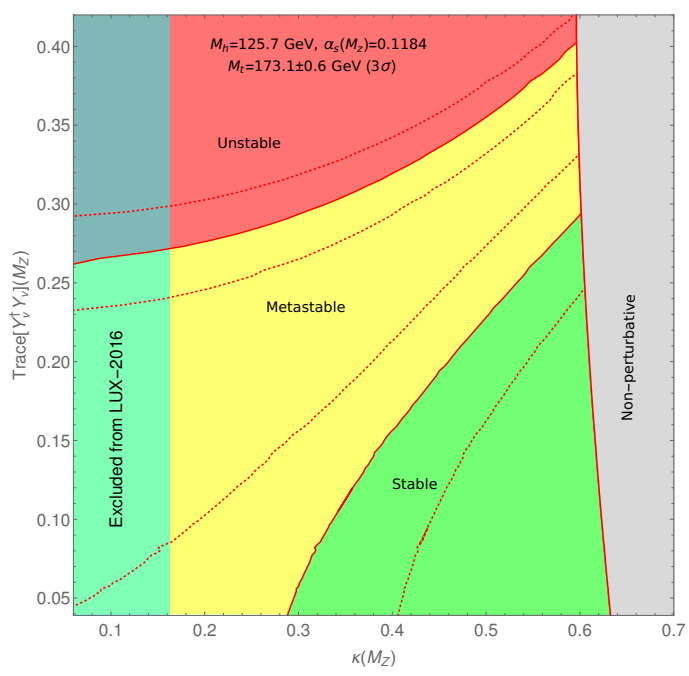

Figure 1: Phase diagram in the $\operatorname{Tr}\left[Y_{v}^{\dagger} Y_{V}\right]-\kappa$ plane. The three boundary lines (two dotted and a solid) correspond to $M_{t}=173.1 \pm 0.6 \mathrm{GeV}(3 \sigma)$. The dark matter mass is dictated by $\kappa\left(M_{z}\right)$ to give the correct relic density. ity while the singlet scalar couplings push the vacuum towards stability. We find that it is possible to find parameter spaces for which the electroweak vacuum remains absolutely stable in the presence of the extra scalar particle. We find an upper bound from metastability on $\operatorname{Tr}\left[Y_{v}^{\dagger} Y_{V}\right]$ as 0.25 for $\kappa=0$, which increases to 0.4 for $\kappa=0.6$. Here, $Y_{v}$ is the Yukawa coupling matrix of the neutrinos. The correlations between the Yukawa couplings $\operatorname{Tr}\left[Y_{v}^{\dagger} Y_{V}\right]$ and $\kappa$ is presented in the phase diagram shown above.

\section{References}

[1] I. Garg, S. Goswami, Vishnudath K.N. and N. Khan, Phys. Rev. D 96, no. 5, 055020 (2017) doi:10.1103/PhysRevD.96.055020 [arXiv:1706.08851 [hep-ph]]. 